\title{
A PERSONAL REVIEW OF THE XXII WORLE CONGRESS ON SPORTS MEDICINE, Vienna, 1982
}

\author{
by PETER SPERRYN, FRCP (Glasg.), DPhysMed., Honorary Secretary, BASM
}

The Vienna World Congress on Sports Medicine in July 1982 highlighted the worldwide spread of interest and research in the subject. Sports medicine is now a multi-disciplinary field covering the whole of exercise, science and medicine. Two pressures mark its advance: the need for greater intra-disciplinary expertise in line with advancing technology and research and the inevitable need for multi-disciplinary co-operation as the complexity of the field increases.

Three main themes ran through the scientific programme. Firstly, mass sport and exercise is becoming a governmentbacked movement, linked with Public Health throughout the world.

Secondly, high performance sport continues to attract top research interest. Together with research into cellular levels of performance, a new technology is developing of movement analysis, e.g. in running and other sports and orthopaedic research is becoming more bio-mechanically orientated.

Thirdly, sports injuries are always a prominent part of such meetings but the quality and quantity of communications has improved almost out of recognition over the past decade. The running revolution has triggered one set of research stimuli - into bio-mechanics, the foot, over-use injuries and footwear, but it is also as if the orthopaedic world had suddenly discovered sport as an outlet for its own newly emerging technology and a profusion of ingenious and original papers on injuries reflected this.

\section{The Knee}

The knee is the more frequently injured joint in sport and a natural focus for communications on the management of a wide range of pathologies.

Of particular interest were Chinese reports of management of patello-femoral lesions, not only by more traditional shaving or drilling of roughened cartilage but by filling articular defects with silicone. Methods and controls are uncertain but the reported good results need to be duplicated urgently.

Others reported good results from intra-articular injection of various substances into the chondromalacic knee. These were all from European centres, both East and West and sound so promising that we may be on the verge of a substantial advance in the management of this universal bugbear of active sportsmen - just imagine the future management of chondromalacia including a simple cartilage-restoring injection to cut short the months of chronic misery suffered by many who have not responded to the ritual straight leg quadriceps contractions or even to orthoses.

Several centres are using CAT scans in knee diagnosis and claim precision in early patello-femoral lesions as well as with cartilage and cruciate damage. Some advocate early correction by surgery of any mechanical instability as a preventive measure against later chondromalacia patellae (CP) or arthritis.

Arthroscopy remains the focus of the surgeons' vision. A Viennese team led by Jelinek and Sellner reviewed its series of 750 sports injury arthroscopies including $\mathbf{5 0}$ arthroscopic meniscectomies and this technique has clearly established its value. Cruciate surgery tantalises surgeons. The generally unsatisfactory results of traditional repairs or of doing nothing - has made centres look at more objective testing of cruciate function with mechanical gauges. Ingenious new repair techniques, including use of reinforcing medial retinacular strips, are still being devised by Renström and colleagues. A challenging review by Johnson from Vermont of North American surgical practice showed that in isolated anterior cruciate tears in fit athletes $30 \%$ of surgeons would treat non-operatively, $65 \%$ would repair with or without grafting, and $7 \%$ would ignore the ligament and would replace it with a graft or reconstruct the joint capsule. Bearing in mind the different techniques with each of these options, you get some ideas of the remaining challenges to be met! It should always be remembered that these figures concern athletically perfect functional results and a non-sportsman probably should not be at too great a disadvantage whatever management is followed.

\section{The Ankle}

The ankle is next in importance with sporting orthopaedists and, as with the knee, basic arguments still concern accurate initial diagnosis. Mann, from San Francisco, gave his usual excellent contribution, correlating functional anatomy with clinical and $X$-ray examination and showed the importance of doing not only plain and neutral talar tilt X-rays but of having tilt views in plantar and dorsi-flexion too, in order to take in the anterior and posterior lesions which are often missed. Peroneal tenography is increasingly used to exclude calcaneo-fibular rupture. Two large studies 
from Maastricht in Holland challenged common diagnostic and therapeutic practices. Firstly, no correlation was found between X-ray findings and the degree of ligament rupture found at operation - "Radiological assessment can do nothing more than indicate presence or absence of connective tissue damage", though arthrography and inversion stress $X$-rays were sensitive indicators of lateral ankle damage, especially stress films under general but not local anaesthesia.

When it came to treatment, a random prospective trial in 150 patients out of a massive series of 921 showed no advantage in early operative repair and/or six weeks plaster immobilisation compared with early mobilisation in a bandage.

At the one year follow-up, all groups were equal for stability, stress $X$-rays and function.

This was agreed by a British paper by Hutson and Jackson advocating early mobilisation with a cast-brace when the ankle was unstable, though others advocated early surgery for laxity. Nilsson, President of the Norwegian Association of Sports Medicine put the case for initial steroid medication and early mobilisation of ankle sprains with quicker recovery than with strapping alone or strapping and ice; and no late disadvantages - perhaps surprising in view of the known evidence for the collagen weakening effect of steroids. So, for practical purposes for most of us, ice and immediate strapping of the sprained ankle is the ideal, followed by as early mobilisation as pain permits. If pain or instability persist, refer for expert help. Certainly it seems difficult to accept some of the claims for early surgery when the late results of conservative management are so favourable. Proprioceptive reeducation, e.g. by the wobble board, is essential for stability and full function.

\section{Prevention of Injuries}

Prevention of sports injuries has to be built into preparation and training. Selection for particular sports remains a teasing problem, but selection out of risk factors may be easier. A study of Swedish Soccer League players by Ekstrand identified over $70 \%$ of injuries as due to preventable factors such as joint instability, muscle tightness or lack of flexibility, inadequate rehabilitation after injuries or neglect of equipment or rules. Systematic correction of these obvious factors succeeded in reducing the injury rate by $75 \%$ compared with control or uncorrected teams. These fundamentals don't need top research facilities or clinicians and bring practical sports medicine into every doctor's daily practice. Greissinger et al from Pittsburgh reported a similar 60\% reduction in injuries in US indoor soccer and a three stage screening programme was offered by van Enst from Amsterdam. The questionnaire was answered by participants who then had a simple locomotor examination by the physiotherapist. Any relevant abnormality was referred to the physician. As a reflection on modern health and fitness, a mean number of three abnormalities per athlete was found.

More specific risks were identified in rugby. A Canadian paper by Schamberger from Vancouver examined the anatomy of cervical movement in the scrum, with particular reference to a case report of severe neurological injury. As with boxing, the inevitable conclusion remains that some sports are inherently - and in the last analysis unpreventably so - dangerous. In the reported case some otherwise minor and unpredictable congenital cervical malformations were crucial in the injury mechanism. A Swiss report from Chur showed head injuries to be common in child skiers and here - as with horse riding - a simple approved head-gear can play a life saving part but public education is needed.

The forces of heading the ball in Soccer was studied with an accelerometer by a team in Munich and results suggested that the average force is much lower than anything encountered in boxing though some small risk of injury remains.

\section{Back Injury}

Back injury remains a difficult clinical problem. One Swiss study also reported a $71 \%$ incidence of spinal X-iay damage in professional riders with a tendency to changes similar to Scheuermann's diseases. No excess of spondylolisthesis was found in his series of 115 mixed ability riders. Another German orthopaedist, Menge from Dusseldorf, had studied adolescent scoliosis with reference to asymmetrical sporting stresses on the back. He concluded that scoliosis was independent of such movements.

Oseid's Norwegian studies of 48 girl gymnasts (average age 14.7) showed a 62.5 incidence of low backache, compared with $75 \%$ in a 1971 study and $22.5 \%$ in matched non-gymnastic controls. Hypermobility and excessive lumbar lordosis was present in $50 \%$ contrasted with only $20 \%$ in controls. Attention was drawn to the fact that the greatest incidence of back injury occurred in those undergoing intensive training to encourage spinal mobility - at the expense of stability - from an early age. Incidentally it was also found that the stiffer the shoulders, the greater the lower back pain due to compensatory hypermobility. No clearer warning can be found for the ambitious pusher of children into the increasingly complex gymnastic movements of today's sport. If your child does not naturally bend 
over double, you should not try and train him to do so. We all have inherent limits on our spinal range, which we transgress only at the cost of pain and perhaps permanent injury.

\section{Injury Management}

A powerful plea came from Northern Europe for special clinics for sport, based on the low pick-up rate prevalent in so many routine casualty departments. Westermann's major study of 1250 sports injuries in Hannover showed marked neglect of early accurate diagnosis in serious ligament lesions, with a consequently worse surgical prognosis. This echoes earlier pleas from many sources, showing a high rate of mis-management in routine hospital departments, especially casualty and orthopaedic clinics without specific sporting experience or interest. Awareness, it seems, is the key to early recognition of the sportsman's lesions, so often partly masked initially by fitness, compensation and plenty of muscle.

Debate continues about the role of sport in late degenerative arthritis. A Munich paper found over-use changes in $\mathbf{8 6 \%}$ of sporting legs, though only half of the patients had ever had symptoms. A Berlin report by Bartsch and Weigert following up professional footballers found knee and ankle OA clearly related with injury, but, by contrast, hip changes reflected underlying malformations and dysplasias - hence another possible indication for screening out high-risk players early in resistant "groin strain" and certainly for examining hip range bilaterally in every case and considering $\mathrm{X}$-rays fairly often.

Soft-tissue injury research looks more interesting nowadays and some fundamental questions are coming under review. Degenerative joint changes in rabbits were shown by a Heidelberg team led by Puhl to be inhibited by injections of polysaccharide or polysulphuric ester. (This ties up with the clinical reports of intra-articular therapy for patellofemoral lesions.)

New diagnostic tools are on the horizon. CAT scanning and arthroscopy have been mentioned. Thermographic studies of soft-tissue lesions were reported from both New York and East Germany, the latter most interestingly in a direct attempt to tally up hot or cold therapy with local temperature findings following injury. Italian workers offered the space age prospect of laser therapy for soft tissues, but again, the results seemed good without the comparison of a controlled trial.

Fowler's elegant study of Canadian-swimmers' styles and their shoulder injuries pinpointed coraco-acromial arch impingement ischaemia as an important factor. A plea by Widhalm of Vienna was made for forearm surgery for tennis elbow - Garden's operation of lengthening extensor carpi radialis brevis rather than attacking the extensor origins themselves at the elbow. Elbow lesions in javelin throwing were comprehensively described by the Finnish doctors Peltokallio, Harjula and Kairento looking after the world's best throwers and over half the men were found to have movement limitations and also dengenerative changes on $\mathrm{X}$-ray.

Here a good style and coaching are absolutely crucial.

\section{Running Advances}

Running has attracted much study. New technology gives rapid computerised analysis of movement patterns, micro techniques permit study of pressure changes within shoes as well as the tissues.

General scientific propaganda for running continues and more selective subgroups have been studied. Six ultra marathoners ran across Germany and, with another longer term cohort study of $\mathbf{3 5 0}$ modestly trained Nuremberg long distance runners over 9 years, added to the bank of baseline information concerning the safety of long term moderate aerobic exercise on the cardiorespiratory system. Also, uric acid and cholesterol levels were lowered. A Roman study of $24 \mathrm{hr}$ ambulatory ECG monitoring of middle-aged (35-52 years) fit marathoners showed a small and harmless incidence of various ectopic beats.

Several bio-mechanical studies showed the researchers' ingenuity. EMG studies on the relative activity of the leg muscles sprinting over a force plate showed that landing was more energetic than take-off, especially at slower speeds. The lesser effort needed in thrust-off gave scope for smoother movement with minimal energy output when cruising one of those tantalising extras which perhaps the greatest runners have which minimises the work of running to give them physiological advantage.

A new concept of posterior compartment pressure symptoms in the thigh was demonstrated by Peltokallio from 
Helsinki. This challenged a lot of preconceptions about cramp and chronic hamstring sprain with its relief of hamstring pain by compartment decompression.

Leg length discrepancy has long been a controversial topic. The standard orthopaedic attitude is that it is unimportant. However. Friberg and Kvist from the Turku Military Hospital and University have correlated symptoms with leg length in $\mathbf{1 4 0 0}$ military recruits of them. The accuracy of their limited-dose radiographic measurements was extreme and reproducible, their anatomical reference points standardised and their conclusions remarkable - that leg length differences of even under 5 millimetres have significant clinical effects. A slightly longer take-off leg was a possible advantage to jumpers - but for runners it is an important aetiological factor for many over-use injuries. We now have a basis for correction of leg differences - BUT SLOWLY, because hasty shoe additions simply cause an acute short-leg syndrome. As it is still true that the majority of people don't develop symptoms, this management mainly concerns the more active sports participant.

Malalignments of the legs are increasingly coming under study. The monumental Vancouver study of runners was reported together with further bio-mechanical studies by its authors. Firstly, electro-goniometry was used to analyse the changes in running style in response to footwear and orthotic changes. Secondly, oxygen consumption was measured with and without orthoses at different running speeds. In the tests done, no changes in oxygen uptake were shown, but this is possibly because exercise level was sub-maximal in intensity as well as duration. Further studies will now look at the prolonged sub-maximal durations relevant to long distance racing. The Nike Laboratories showed increasing energy expenditure as running shoes got heavier.

\section{Fitness for the Masses}

Keynote addresses were given by Kuroda of Tokyo and Strauzenberg (Dresden), President of FIMS' Scientific Commission. Each was concerned with older age normal men, not athletes, who continued regular fitness programmes based on endurance running and jogging. Good adaptation to exercise stress was found at all ages - but with rapid loss of benefit if the programme was stopped. Biochemical markers of fitness - cholesterol, lipids and urate - improved and blood pressure diminished - probably through reduction in adrenergic, or sympathetic drive. The pre-condition for improvement is regularity, and while these programmes used up to five sessions a week, it is accepted from other studies that a minimum of $2-3$ days a week is needed.

An important selling point for exercise is safety. The Finns Vuroi and colleagues outlined a "cost benefit" case for exercise programmes. You need to compare exercise-induced death rates with expected or inevitable deaths and the demonstrable effects of "death postponement" through better health from exercise. (For instance, society might find it cheaper to accept early lazy death than to pay for all the sport and exercise if the latter started escalating health costs through injuries and increased medical demands, e.g. for fitness screening and induced coronaries!) The welter of statistics available all point to net health and economic benefits of a healthier way of life, though the effect of exercise, by itself, remains uncertain because of the complex inter-relation of all the various health-and risk-factors.

Nevertheless, more and more centres have established safe and enjoyable programmes. Jogging, running, cycling, walking, swimming will all do, but violent sport and heavy weight training won't because they do not induce the same cardio-respiratory responses of aerobic sub-maximal exercise, and they may have a clear risk of myocardial infarction related to sudden hypertension. A team from Florence led by Attina demonstrated the advantages of such programmes on the systolic BPs of older subjects; another from Bratislava demonstrated life-long performance levels in steady state events.

\section{Biochemical Changes}

Bone mineral density was shown to decline gradually with age in a sample of 138 Finnish men, but the age-decline was delayed by physical activity. This finding is of considerable importance, suggesting yet another way in which regular exercise delays ageing changes in tissues. Recent reports of de-mineralisation in female athletes should be studied very critically in the light of the overall published data.

Blood pressure responses to exercise are still being elucidated. While the general tendency of long-term aerobic exercise is to lower BP levels, some new details of controls are emerging, which may have clinical significance in patient management. A NASA team from Houston showed that age was related independently to resting and exercising BP levels. Thus each patient must be individually assessed for exercise-BP variation. An Italian study concluded that borderline hypertensives should be treated as hypertensive patients and not as normals, and therefore should be excluded from maximal exercise. 
Exercise-BP responses after myocardial infarction have been extensively studied, particularly in the North American programmes which established the safety of fairly severe training programmes. A Norwegian extended this work to long distance ski-racing, with $30 \%$ improvement in work capacity shown in the month-long post-coronary training programme, leading to successful racing at a level of $80-85 \%$ of maximum capacity. The authors drew attention to the specific problem of cold intolerance which adds a new dimension of risk to the lives of snow-dwelling anginals.

Therapeutic advances may create new problems and beta-blockers remain an important research topic. These drugs vary greatly in their individual effects, as do patients in their responses. The most important under-publicised effect of some beta-blockers is to interfere with carbodhydrate mechanism and can cause prolonged and distressing hypoglycaemia, resistant to the usual sugar intake. This should be explained to patients where there is a chance of prolonged exercise sessions. The remedy is most likely to be a switch of drug.

\section{Anaemia in Exercise}

Apparent anaemia may occur in heavy training. Some athletes happen to have a nutritional anaemia and a large proportion of women combine under-eating with menstrual losses which will deplete iron stores and lower haemoglobin. They need accurate diagnosis, then iron. Training often leads to increased haemoglobin and erythrocyte production but also a physiological haemodilution so that the tested haemoglobin may appear to fall. There is no anaemia. No iron is needed and the iron stores are normal. If only coaches could grasp this point! The health of female athletes has been much studied lately and bone mineral changes, menstrual dysfunction and hormonal changes as well as weight loss have been described. Oseid's large study of Norwegian international level sportswomen revealed hormonal changes of simple hypothalamic suppression, presumably due to training stress on average over 16 hours weekly. Androgen levels were normal, possibly because of altered hormonal conversion in the much diminished body fat, which normally plays a greater part in androgen metabolism. Top Polish girl swimmers showed frequent late menarche (one in six) and menstrual disorders (43\%). These authors recorded concern about the medical consequences, including chronic respiratory infection and musculo-skeletal pains, in this young and vulnerable group due to intensive training.

Dutch studies of the differences in onset of puberty in relation to body weight and fat content in swimmers and gymnasts were inconclusive, but both European and American studies are proceeding on this important topic. It may be that we shall find that there are fine limits of training intensity beyond which an increasing and possibly unacceptable proportion of our youngsters may develop quite complex hormonal disorders affecting growth and health itself. We should watch this topic very carefully.

\section{Adolescence}

Adolescents are always vulnerable. Much work on the adolescent athlete has been published and in addition to the menstrual problems Oseid delineated the incidence of back disorders in groups of girl gymnasts aged 15 in 1981, compared with a similar group in 1971, and a control group. The incidence of low back pain ranged from over $60 \%$ in gymnasts to only $22.5 \%$ in controls. More worrying still, the group found $44 \%$ of the earlier group to have permanent back symptoms (controls 2.5\%). The greatest incidence of back injury was in young gymnasts under intensive spinal mobility training. A clue to back trouble may be found in the shoulders. Stiff shoulders have to be compensated by extra lordosis or movement in the young gymnast's spine and thus spinal injury occurs. More detailed coaching attention to non-spinal mobility should pay dividends.

Menge found no evidence for a relationship between idiopathic scoliosis and sports movement - intensive unilateral training movements in adolescence do not cause this condition. However, a Swiss survey showed a high incidence of spinal degenerative changes due to riding, ranging from an overall incidence of $17.5 \%$ of all 115 riders up to $71 \%$ in the professional riders. Half the riders had backache, but the $4 \%$ incidence of spondylolisthesis was compatible with control levels.

\section{Limits of Performance}

What stops us? As we unravel more of the mysteries of human performance physiology we are drawn away from the question "How do we?" towards the fascination of "Why can't we?" The World Congress reflected this trend and a number of quite new ideas and research thrusts were introduced.

Many had drawn attention to the genetic determinants of performance - "Choose your parents", but proving differences between inherited and acquired attributes can be difficult. The German National Football Squads, both senior and youth, showed marked increases in all physiological tests between 1974 and 1981, presumably reflecting both selection (of the gradual trend towards bigger people) and more refined conditioning of select squads (Nowacki and 
de Castro). Another ingenious experiment by Daniels from the Nike Laboratories sought to compare muscular working capacity in different muscle groups. Thirteen trained runners were put through different efforts of running, cycling, walking on gradients, stepping up and down and arm-cranking. Running ability did not correlate with the other modes of exercise even after training improvements. The hypothesis was that exercise - economy may be predetermined genetically, though variably modifiable with training. Even this leads to further doubts. An EMG study of motor unit potentials, reflecting active contraction, showed that three groups of power sportsmen - boxers, judoists and weightlifters differed both from each other and from controls. It could be that they each had subtle differences in innate endowment of muscle-fibre chemistry or, by contrast, that the subtle differences may have been in training and movement techniques (Nonsianen et al from Kuopio). After all the high science of recent years, several laboratories have been looking for simple translations of exercise tests into cheap day to day methods for the coach or physician. I think we will see a move back to simplicity - such as simple pulse counts in simple step tests - with the complex tests confirming the general validity and correlations of such tests. We need to remember the basic question "What am I trying to test"?

\section{Over-Training}

An ingenious study by Verstappen from Holland showed daily variation in work capacity "indicating a physiological rather than psychological basis" for those daily fluctuations in form so long known but ill-understood by coaches and athletes alike. Why do you feel - and compete - so variably from day to day despite the best planned training schedules?

Research papers are beginning to emphasise metabolic factors rather more than the older topics of oxygen transport and muscle contraction. Hormonal and mineral limits are being defined. Fatigue itself is a poorly understood final common pathway of many metabolic sequences. The mechanism was explored in a paper from Copenhagen which related to the lactate production in explosive events. With acid-induced slowing of glycolysis in endurance events, substrate depletion becomes a limiting factor, but common to both types of exercise may be potassium and water shifts through the cell membranes which finally impair muscle-fibre contraction.

In looking at such cellular mechanisms, special training methods and fluid replacement techniques become important. The carbohydrate-loading technique was reviewed by Costill and Shephard's study of sweat composition demonstrated an active regulatory mechanism which could selectively vary the mineral loss in relation to environment and overall sweat flow rate. An understanding of metabolic losses is crucial to sensible replacement, especially in the face of so many claims surrounding commercial sports drinks. One trial simulated tennis conditions over two hours with players taking either carbohydrate drinks, or water, or no fluid replacement. The sugared players maintained skill, power and body weight. This confirms many earlier trials but is valid only in such intermittent plays as tennis, soccer, etc. The marathoner's attempt to energise his progress is frustrated by gastric stasis associated with all but the most dilute fluids and some eminent authorities therefore recommend water alone for such races.

Hormonal studies included those on testosterone and its precursors, growth hormone and the female hormones in relation to the menarche and training. Numerous studies of catecholamines in relation to exercise, hypertension and beta-blockers are slowly unravelling more mysteries and exercise-induced insulin responses are seen as useful adjuncts to diabetic control.

Lurking behind some of these complex endocrine studies is the major conundrum of over-training. What is this, and the "over-use state" and how does it relate to staleness?

Russian interest in the field is evident from a number of papers on health, fitness, over-use breakdown, nervous system over-training and physiological limits. This tallies with the other papers looking at over-use injuries and the clinical problems of highly trained adolescents. We are now looking seriously at human limitations and you can be sure that top sports limits are being defined with increasing anxiety, particularly in Eastern Europe.

Biochemical markers of over-training include hormonal changes and enzyme levels previously associated with frank tissue damage but now thought to reflect stress-induced increases in cell membrane permeability and evidence of myopathy and myoglobin leakage. Muscle soreness, or stiffness, is still not clearly defined - even in the era of heart transplantation!

A small group of tired athletes may have mitral valve prolapse. A four-year Roman study by Venerando's team indicated that although this condition is almost always benign, sufferers tend to have more palpitations, arrhythmias and fatigue than control subjects. Psychological factors limiting sport are well-known. Papers were presented on the 
"Training Champion" phenomenon, "the guy who does it all in training but cannot race" - and on relaxation techniques, particularly the autogenic training so popular in Europe.

Finally the World Congress is, of course, the major four-yearly reunion of the International Federation of Sports Medicine and its major business meetings are important focal points for policy developments. The Inter-Federal Medical Commission, which co-ordinates FIMS with the international governing bodies of sport formally called for wider educational programmes in sports medicine, more co-ordination between authorities, particularly to standardise and to improve dope control protocol and for a more prominent voice for the medical viewpoint in sport, particularly in the drafting of safety regulations.

C Dr. P. N. Sperryn

\section{CORRESPONDENCE}

37 Upper Gordon Road, Camberley, Surrey

To the Editor:

Dear Sir,

\section{NON-STEROIDAL ANTI-INFLAMMATORY DRUGS AND ETHANOL}

In September you published my case report about possible damage to the caecal wall of a marathon runner (Porter, 1982). I now believe it to be an incomplete statement.

In April an important paper was published in the USA (Deykin et al, 1982). The authors found an ethanol-induced potentiation of the effects of aspirin on the bleeding time and a similar potentiation with ibuprofen or indomethacin.

The runner who was the subject of $\mathrm{my}$ report took $100 \mathrm{mg}$ of flurbiprofen (Froben) on the evening before the marathon. In the evening after the marathon he took a modest amount of alcohol. I suspect that there was a drug interaction and that a haematoma of the damaged caecal wall developed. It has been subsequently shown, however, that the subject's bleeding time does not seem to be prolonged by taking alcohol after flurbiprofen. Perhaps extreme exertion is also required to trigger the interaction. The subject will run a further marathon in 1983 repeating the suspect sequence (Froben - exertion - alcohol) and detailed pathological tests will then be done.

Meanwhile I think the work of Deykin et al should be more widely known. It has an obvious importance for all marathon runners and for those who take part in combative sports.

$$
\text { Yours sincerely, }
$$

$$
\text { A. M. W. Porter }
$$

\section{REFERENCES}

Deykin, D., Janson, P., McMahon, L., 1982 "Ethanol potentiation of aspirin-induced prolongation of the bleeding time". The New England Journal of Medicine 306: 852-854.

Porter, A. M. W., 1982 "Marathon running and the caecal slap syndrome". British Journal of Sports Medicine 16: 178. 